\title{
Numerical Solution of Fractional Order Anomalous Subdiffusion Problems Using Radial Kernels and Transform
}

\author{
Muhammad Taufiq and Marjan Uddin \\ University of Engineering and Technology Peshawar, Department of Basics Sciences and Islamiat, Peshawar, Pakistan \\ Correspondence should be addressed to Marjan Uddin; marjan@uetpeshawar.edu.pk
}

Received 13 March 2021; Accepted 3 May 2021; Published 9 July 2021

Academic Editor: Ahmet Ocak Akdemir

Copyright ( 2021 Muhammad Taufiq and Marjan Uddin. This is an open access article distributed under the Creative Commons Attribution License, which permits unrestricted use, distribution, and reproduction in any medium, provided the original work is properly cited.

By coupling of radial kernels and localized Laplace transform, a numerical scheme for the approximation of time fractional anomalous subdiffusion problems is presented. The fractional order operators are well suited to handle by Laplace transform and radial kernels are also built for high dimensions. The numerical computations of inverse Laplace transform are carried out by contour integration technique. The computation can be done in parallel and no time sensitivity is involved in approximating the time fractional operator as contrary to finite differences. The proposed numerical scheme is stable and accurate.

\section{Introduction}

In the last decades, many researchers have studied the fractional calculus [1-3]. Differential equations of fractional order have many applications in the field of science and engineering [4-7]. Analytical solution of many fractional differential equations is not possible or very hard to find, so we need a new numerical technique to find its approximate solution. Various phenomena in viscoelastic materials, economics, chemistry, finance, control theory, hydrology, physics, cosmology, solid mechanics, bioengineering, statistical mechanics, and control theory can be mathematically modeled from fractional calculus [8-17]. In literature, various numerical approaches are available for modeling anomalous diffusive behavior such as Carlo simulations [18]. An introduction of diffusion equations can be found in [19-21].

Recently, RBF-based methods were used in solving fractional partial differential equations (FPDEs) [22-24]. These methods have been employed in approximation of partial differential equations with complex domains. An implicit meshless technique based on the radial basis functions for the numerical simulation of the anomalous subdiffusion equation can be found in [25]. The convergence and stability of these mesh-free methods can be found in $[26,27]$. These globally defined RBF methods cause illcondition system matrices [28]. To overcome the problem of ill-conditioning, local RBF techniques were used in [29-31]. Unlike global RBF methods, the RBF method in local setting uses center points in each subdomain area of influence, surrounding each spatial point due to which there is reduction in the computational cost.

Recently, Laplace transform is combined with RBF method in [32, 33]. In [34-37], the authors use Laplace transform as tool in spectral method and other mesh-based methods such as finite element methods and finite difference method. To avoid the issues of computational efficiency and instability of the system matrix, we introduce a new technique Laplace transform-based local RBF method in solving the time fractional modified anomalous subdiffusion equations in irregular domain.

Here, we consider the following modified anomalous subdiffusion equation of fractional order [38]:

$$
\frac{\partial w(\mathbf{x}, t)}{\partial t}=\left[\nu_{1} D_{t}^{(1-\alpha)}+v_{2} D_{t}^{(1-\beta)}\right] \Delta w(\mathbf{x}, t)+f(\mathbf{x}, t)
$$


where $\mathbf{x}=(x, y) \in \Omega \subset \mathbb{R}^{d}, d \geq 1, t>0$, subject to the following boundary and initial conditions:

$$
\begin{aligned}
\mathscr{B} w(\mathbf{x}, t) & =h(\mathbf{x}, t), \\
\mathbf{x} & =(x, y) \in \partial \Omega, \\
w(\mathbf{x}, 0) & =w_{0}, \quad \mathbf{x} \in \Omega,
\end{aligned}
$$

respectively, where $\alpha, \beta \in(0,1), t \in[0, T], \nu_{1}, v_{2}$ are positive constants, $\Delta$ is the Laplace operator, and $f(\mathbf{x}, t)$ is some given function.

\section{Preliminaries}

Here, we introduce some fundamental definitions related to fractional calculus [39, 40].

Definition 1. Let $n-1<\alpha<n \in Z^{+}$and $\alpha>0$, then the Caputo derivative of fractional order is defined as

$$
D_{t}^{\alpha} w(t)=\frac{1}{\Gamma(n-\alpha)} \int_{0}^{t} \frac{1}{(t-z)^{\alpha+1-n}} \frac{\mathrm{d}^{n}}{\mathrm{~d} z^{n}} w(z) \mathrm{d} z .
$$

Definition 2. Let $w(t), t \geq 0$, be a given function, then its Laplace transform is defined by

$$
\widehat{w}(z)=\mathscr{L}\{w(t)\}=\int_{0}^{\infty} e^{-z t} w(t) \mathrm{d} t,
$$

provided this integral converges.

Lemma 1. If $w(t) \in C^{p}[0, \infty)$, with $\alpha \in(n-1, n) \in Z^{+}$, then the Laplace transform of the fractional order Caputo derivative is given by

$$
\mathscr{L}\left\{D_{t}^{\alpha} w(t)\right\}(z)=z^{\alpha} \widehat{w}-\sum_{i=0}^{n-1} z^{\alpha-i-1} w^{(i)}(0) .
$$

Theorem 1. the Bromwich inversion theorem [41]). Let $w(t)$ have a continuous derivative and let $|w(t)|<K e^{\gamma t}$, where $K$ and $\gamma$ are positive constants. Define

$$
\widehat{w}(z)=\int_{0}^{\infty} e^{-z t} w(t) \mathrm{d} t, \quad \operatorname{Re}(z)>\gamma,
$$

then

$$
w(t)=\frac{1}{2 \pi i} \int_{\xi-i \infty}^{\xi+i \infty} \widehat{w}(z) e^{z t} \mathrm{~d} z
$$

\section{Description of the Method}

3.1. Time Discretization. Here, we apply Laplace transform to models (1)-(3) which gives

$$
\begin{aligned}
{\left[\left(z I-\left(v_{1} z^{1-\alpha}+v_{2} z^{1-\beta}\right)\right)\right] \widehat{w}(\mathbf{x}, z) } & =w(\mathbf{x}, 0) \\
-\left(v_{1} z^{-\alpha}+v_{2} z^{-\beta}\right) \Delta w(\mathbf{x}, 0)+\widehat{f}(\mathbf{x}, z), & \mathbf{x}=(x, y) \in \Omega \subset \mathbb{R}^{d}, \\
\mathscr{B}(\widehat{w}(\mathbf{x}, z))=\widehat{h}(z), \quad \mathbf{x} & =(x, y) \in \partial \Omega .
\end{aligned}
$$

In more compact form, we have

$$
\begin{array}{cc}
\mathscr{L}(\widehat{w}(\mathbf{x}, z))=\widehat{g}(\mathbf{x}, z), \quad \mathbf{x} \in \Omega, \\
\mathscr{B}(\widehat{w}(\mathbf{x}, z))=\widehat{h}(\mathbf{x}, z), \quad \mathbf{x} \in \partial \Omega .
\end{array}
$$

The transformed problems (10) and (11) will be solved for the solution $\widehat{w}(\mathbf{x}, z)$ using local RBF method. The solution $w(\mathbf{x}, t)$ of the given models (1)-(3) will be found by using numerical inversion.

3.2. Local Radial Basis Functions Method. Here, the linear operators $\mathscr{B}$ and $\mathscr{L}$ are discretized by using local RBF [42, 43]. Consider the centers $\left\{x_{i}, i=1 \ldots, N\right\} \subset \Omega \subset \mathbb{R}^{d}, d \geq 1$, where $\Omega$ is the bounded domain. For each point $x_{i}, i=1,2,3, \ldots, N$, we can find a subdomain $\Omega_{j}$ such that $n<N$. The unknown function $\widehat{w}(\mathbf{x}, t)$ can be approximated with RBF in each local subdomain $\Omega_{i}, i=1,2, \ldots, N$, by the following equation:

$$
w\left(\mathbf{x}_{i}, t\right) \approx \widehat{w}\left(\mathbf{x}_{i}, t\right)=\sum_{j=1}^{n} \lambda_{i_{j}}^{i} \phi^{i}\left(\left\|\mathbf{x}_{i}-x_{j}\right\|\right), \quad x_{j} \in \Omega_{j},
$$

where $\lambda^{i}=\left[\lambda_{1}^{i}, \lambda_{2}^{i}, \ldots, \lambda_{n}^{i}\right]$ are the unknown coefficients, and $r_{i j}=\left\|\mathbf{x}_{i}-x_{j}\right\|$ is the norm between nodes $\mathbf{x}_{i}$ and $x_{j}, \phi(r)$, $r \geq 0$ is a radial kernel (multiquadric radial basis function), and $\Omega_{j} \subset \Omega$ is a local domain for around each $\mathbf{x}_{i}$, containing $n$ neighboring nodes around the node $\mathbf{x}_{i}$. So, we have $N$ small size linear systems each of order $n \times n$ given by

$$
\left(\begin{array}{c}
\widehat{w}_{1}^{i} \\
\widehat{w}_{2}^{i} \\
\vdots \\
\widehat{w}_{n}^{i}
\end{array}\right)=\left(\begin{array}{cccc}
\phi_{11}^{i} & \phi_{12}^{i} & \ldots & \phi_{1 n}^{i} \\
\phi_{21}^{i} & \phi_{22}^{i} & \ldots & \phi_{2 n}^{i} \\
\vdots & \vdots & \ddots & \vdots \\
\phi_{n 1}^{i} & \phi_{n 2}^{i} & \ldots & \phi_{n n}^{i}
\end{array}\right)\left(\begin{array}{c}
\lambda_{1}^{i} \\
\lambda_{2}^{i} \\
\vdots \\
\lambda_{n}^{i}
\end{array}\right), \quad i=1,2, \ldots, N,
$$

which can be denoted by

$$
\widehat{\mathbf{W}}^{i}=\mathbf{S}^{i} \lambda^{i}, \quad i=1,2, \ldots, N,
$$

where $\phi_{j k}^{i}=\phi^{i}\left(\left\|x_{i_{j}}-x_{i_{k}}\right\|\right), x_{i_{j}}, x_{i_{k}} \in \Omega_{i}$, and matrix $\mathbf{S}^{i}$ is the system matrix.

Now, applying the operator $\mathscr{L}$ to (12) gives

$$
\mathscr{L} \widehat{w}\left(x_{i}\right)=\sum_{j=1}^{n} \lambda_{i_{j}}^{i} \mathscr{L} \phi^{i}\left(\left\|x_{i}-x_{i_{j}}\right\|\right), \quad x_{i_{j}} \in \Omega_{i} .
$$

The vector form of (15) is given by

$$
\mathscr{L} \widehat{w}\left(x_{i}\right)=\mathbf{G}^{i} \cdot \lambda^{i},
$$

where $\mathbf{G}^{i}$ is given by 


$$
\mathrm{G}^{i}=\mathscr{L} \phi^{i}\left(\left\|x_{i}-x_{i_{j}}\right\|\right), \quad x_{i}, x_{i_{j}} \in \Omega_{i} .
$$

From equation (14), the unknown coefficients $\lambda^{i}$ are given by

$$
\lambda^{i}=\left(\mathbf{S}^{i}\right)^{-1} \widehat{\mathbf{W}}^{i},
$$

and by inserting the values of $\lambda^{i}$ in (16), we have

$$
\mathscr{L} \widehat{w}\left(x_{i}\right)=\mathbf{G}^{i}\left(\mathbf{S}^{i}\right)^{-1} \widehat{\mathbf{W}}^{i}=\mathbf{N}^{i} \widehat{\mathbf{W}}^{i},
$$

where

$$
\mathbf{N}^{i}=\mathbf{G}^{i}\left(\mathbf{S}^{i}\right)^{-1} .
$$

Hence, the discretized form is given by

$$
\mathscr{L} \widehat{w} \equiv \mathbf{H} \widehat{\mathbf{W}},
$$

where matrix $\mathbf{H}$ is called the sparse differentiation matrix of order $N \times N$.

\section{Numerical Inversion Technique}

In this section, the numerical inversion of Laplace transform for approximating the given models (1)-(3) is as follows:

$$
\begin{aligned}
w(\mathbf{x}, t) & =\frac{1}{2 \pi \imath} \int_{\tilde{\xi}-\iota \infty}^{\tilde{\xi}+\iota \infty} \widehat{w}(\mathbf{x}, z) e^{z t} \mathrm{~d} z \\
& =\frac{1}{2 \pi \imath} \int_{\Psi} e^{z t} \widehat{w}(\mathbf{x}, z) \mathrm{d} z, \quad \tilde{\xi}>\widetilde{\xi}_{0},
\end{aligned}
$$

where $\Psi$ is the suitable path joining $\widetilde{\xi}-\iota \infty$ to $\widetilde{\xi}+\iota \infty$. This Bromwich integral is numerically solved by using the following hyperbolic contour [37]:

$$
z(\widetilde{\eta})=\omega+\widetilde{\lambda}(1-\sin (\widetilde{\sigma}-\widetilde{\imath})), \quad \text { for } \widetilde{\eta} \in R,
$$

with $\tilde{\lambda}>0, \omega \geq 0,0<\widetilde{\sigma}<\tilde{\beta}(1 / 2) \pi$, and $(1 / 2) \pi<\tilde{\beta}<\pi$.

Integral in (22) gives

$$
w(\mathbf{x}, t)=\frac{1}{2 \pi \imath} \int_{-\infty}^{\infty} e^{z \widetilde{\eta}) t} \widehat{w}(\mathbf{x}, z(\widetilde{\eta})) \dot{z}(\widetilde{\eta}) \mathrm{d} \tilde{\eta} .
$$

Next applying trapezoidal rule for approximation of (24), we have

$$
w_{k}(\mathbf{x}, t)=\frac{k}{2 \pi \iota} \sum_{j=-M}^{M} \widehat{w}\left(\mathbf{x}, z_{j}\right) e^{z_{j} t^{\prime}} z_{j}, \quad z_{j}=z\left(\widetilde{\eta}_{j}\right), \widetilde{\eta}_{j}=j k,
$$

where $k$ is the step size.

\section{Application of the Method}

In this section, the proposed numerical scheme is applied to multidimensional problems. We solved four test problems and used various domain points $N \in \Omega$, stencils points $n \in \Omega_{j}$, and quadrature points $M$. Three error formulas, the error estimate, $L_{\text {est }}=e^{(-c M / \log (M))}, L_{\infty}$, and $L_{2}$ norms are used. The radial kernel used in our computations is $\phi(r, \varepsilon)=\sqrt{1+\varepsilon^{2} r^{2}}$. The shape parameter $\epsilon$ is optimized by the uncertainty rule related to RBFs.

Problem 1. Consider models (1)-(3) to the following form [38]:

$$
\begin{aligned}
& \qquad \frac{\partial w(x, t)}{\partial t}=\left(D_{0 t}^{1-\alpha}+D_{0 t}^{1-\beta}\right)\left[\frac{\partial^{2} w(x, t)}{\partial x^{2}}\right]+f(x, t), \\
& \qquad f(x, t)=\exp (x)\left[(1+\alpha) t^{\alpha}-\frac{\Gamma(2+\alpha+\beta)}{\Gamma(1+2 \alpha+\beta)} t^{2 \alpha+\beta}\right. \\
& \text { wing boundary and initial conditions: } \\
& \begin{array}{l}
w(0, t))=t^{(1+\alpha+\beta)}, \\
w(1, t)=e t^{(1+\alpha+\beta)}, \quad 0<t \leq T, \\
w(x, 0)=0, \quad x \in(0,1),
\end{array}
\end{aligned}
$$$$
f(x, t)=\exp (x)\left[(1+\alpha) t^{\alpha}-\frac{\Gamma(2+\alpha+\beta)}{\Gamma(1+2 \alpha+\beta)} t^{2 \alpha+\beta}-\frac{\Gamma(2+\alpha+\beta)}{\Gamma(1+\alpha+2 \beta)} t^{\alpha+2 \beta}\right],
$$

with the following boundary and initial conditions:

$$
\begin{aligned}
& \lambda=\frac{\theta r_{b} N}{b T}, \\
& b=\cosh ^{-1}\left(\frac{1}{\theta \tau \sin (\sigma)}\right), \\
& \omega=2,
\end{aligned}
$$

respectively, where the actual solution is given by

$$
w(x, t)=\exp (x) t^{1+\alpha+\beta} .
$$

In our numerical scheme, we used the hyperbolic contour (23). The optimal parameter values are taken as$$
\sigma=0.3812,
$$$$
x_{k}=h k,
$$

$r_{b}=2 \pi r, r=0.3431, h=b / N, \tau=t_{0} / T, t_{0}=0.5, t=1$, and $T=5$. This test problem is solved in the domain $(0,1)$. Here, 
the number of points in domain $\Omega$ is denoted by $N$, the points in local subdomain $\partial \Omega_{j}$ are denoted by $n$, and the number of quadrature points relates to $M$. The numerical solutions are shown in Table 1 with various values of fractional order $\alpha$ and $\beta$ and nodal points $N$. For comparatively smaller values of fractional order $\alpha$ and $\beta$, better results in terms of $L_{\infty}$ and $L_{2}$ error norms are obtained. In the upper part of Table 1, condition number increases, as we increase nodal points $N$. Error versus various quadrature points $M$ at $N=21, n=9$, and $t=1$ and various values of $\alpha$ and $\beta$ are shown in Figure 1. The error estimate $L_{\text {est }}$ for $c=1$ is well matched with $L_{\infty}$ and $L_{2}$ error norms, as shown in Figure 1. Hence, our proposed method is stable and accurate.

Problem 2. Consider models (1)-(3) corresponding to the form [38]
TABLE 1: Numerical results using the proposed numerical scheme corresponding to Problem 1.

\begin{tabular}{lccc}
\hline$M=50, n=7$ & \multicolumn{3}{c}{$\alpha=0.2, \beta=0.1$} \\
\hline$N$ & $L_{\infty}$ & $L_{2}$ & $\kappa$ \\
11 & $8.9603 \mathrm{e}-004$ & 0.0021 & $2.2091 \mathrm{e}+021$ \\
21 & $8.5582 \mathrm{e}-004$ & 0.0028 & $9.6329 \mathrm{e}+021$ \\
31 & $8.4544 \mathrm{e}-004$ & 0.0034 & $2.2821 \mathrm{e}+022$ \\
41 & $8.8689 \mathrm{e}-004$ & 0.0041 & $4.4923 \mathrm{e}+022$ \\
51 & $8.6646 \mathrm{e}-004$ & 0.0045 & $7.5954 \mathrm{e}+022$ \\
71 & $9.2962 \mathrm{e}-004$ & 0.0057 & $1.6860 \mathrm{e}+023$ \\
\hline$M=50, n=7, N=21$ & $L_{\infty}$ & $L_{2}$ & $\kappa$ \\
\hline$(\alpha, \beta)=(0.2,0.6)$ & 0.0141 & 0.0462 & $1.0025 \mathrm{e}+021$ \\
$(\alpha, \beta)=(0.2,0.4)$ & 0.0072 & 0.0236 & $3.6776 \mathrm{e}+020$ \\
$(\alpha, \beta)=(0.2,0.1)$ & $8.5582 \mathrm{e}-004$ & 0.0028 & $9.6329 \mathrm{e}+021$ \\
$(\alpha, \beta)=(0.6,0.3)$ & 0.0079 & 0.0258 & $3.1036 \mathrm{e}+020$ \\
$(\alpha, \beta)=(0.4,0.3)$ & 0.0063 & 0.0207 & $3.1036 \mathrm{e}+020$ \\
$(\alpha, \beta)=(0.1,0.3)$ & 0.0035 & 0.0116 & $9.2023 \mathrm{e}+021$ \\
\hline
\end{tabular}

$$
\begin{aligned}
\frac{\partial w(x, t)}{\partial t} & =\frac{1}{2}\left(\frac{\partial^{1-\alpha} w(x, t)}{\partial t^{1-\alpha}}+\frac{\partial^{1-\beta} w(x, t)}{\partial t^{1-\beta}}\right)\left[\frac{\partial^{2} w(x, t)}{\partial x^{2}}\right]+f(x, t), \\
f(x, t) & =\exp (x)\left[(1+\alpha) t^{\alpha}-\frac{\Gamma(2+\alpha)}{\Gamma(1+2 \alpha)} t^{2 \alpha}+(1+\beta) t^{\beta}-\frac{\Gamma(2+\beta)}{\Gamma(1+2 \beta)} t^{2 \beta}\right],
\end{aligned}
$$

initial and boundary conditions given by

$$
\begin{aligned}
& w(x, 0)=0, \quad x \in(0,1), \\
& w(0, t)=t^{(1+\alpha)}+t^{(1+\beta)}, \\
& w(1, t)=\exp (1)\left(t^{(1+\alpha)}+t^{(1+\beta)}\right), \quad 0<t \leq 1 .
\end{aligned}
$$

The actual solution is

$$
w(x, t)=\exp (x)\left(t^{(1+\alpha)}+t^{(1+\beta)}\right) .
$$

The same domain and same parameter values as used in Problem 1 are incorporated. The numerical results are shown in Table 2 with the same as well as with various values of fractional order $\alpha$ and $\beta$ and nodal points $N$. For comparatively identical values of fractional order $\alpha$ and $\beta$, better results in terms of $L_{\infty}$ and $L_{2}$ error norms are obtained. In the upper part of Table 2, condition number of the system matrix is fixed for $11 \leq N \leq 71$. Error versus various quadrature points $M$ at $N=41, n=9$, and $t=1$ and various values of $\alpha$ and $\beta$ are depicted in Figure 2. The error estimate $L_{\text {est }}$ for $c=0.7$ is well agreed with $L_{\infty}$ and $L_{2}$ error norms, as shown in Figure 1. The results obtained by our proposed numerical scheme are comparatively identical with the results in Table 2 [38].

Problem 3. Next, we consider models (1)-(3) corresponding to the form [44]

$$
\frac{\partial w(x, y, t)}{\partial t}=\left(D_{0 t}^{(1-\alpha)}+D_{0 t}^{(1-\beta)}\right)\left[\frac{\partial^{2} w(x, y, t)}{\partial x^{2}}+\frac{\partial^{2} w(x, y, t)}{\partial y^{2}}\right]+f(x, t)
$$

where

$$
\begin{array}{r}
f(x, y, t)=2 t \sin (2 \pi x) \sin (2 \pi y) \\
\left(1+\frac{8 \pi .^{2}}{\Gamma(2+\alpha)} t^{\alpha}+\frac{8 \pi .^{2}}{\Gamma(2+\beta)} t^{\beta}\right),
\end{array}
$$

initial and boundary conditions given by

$$
\begin{aligned}
w(x, y, 0) & =0, \quad x, y \in \Omega, \\
w(0, t) & =0, \\
w(1, t) & =0, \quad t>0 .
\end{aligned}
$$

The exact solution is

$$
w(x, y, t)=t^{2} \sin (2 \pi x) \sin (2 \pi y)
$$



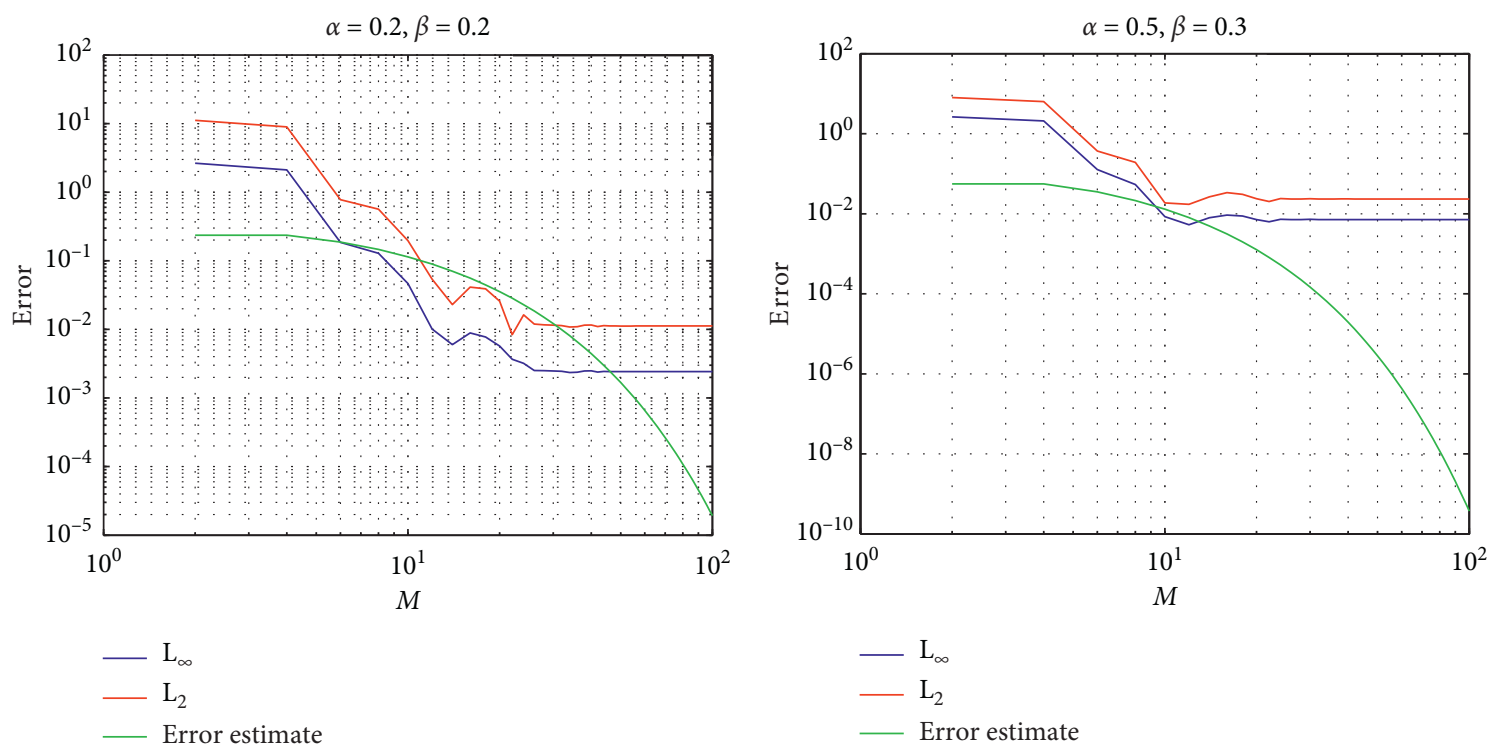

(a)

(b)

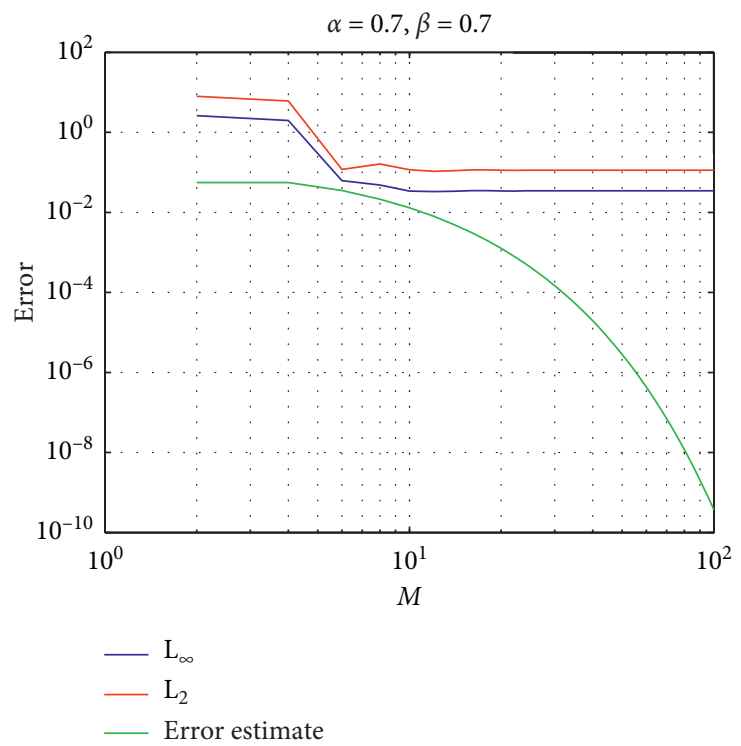

(c)

Figure 1: Error versus quadrature points $M$ at $t=1$ and various values of $\alpha, \beta$ corresponding to Problem 1 .

This problem is solved over the domain $\Omega=[0,1]$. In Table 3, for various nodal points $N$ and stencils points $n=$ 11,15 and with various values of $\alpha$ and $\beta$, the $L_{\infty}$ error norm is well matched with $L_{2}$ error norm. The condition number is increasing steadily as we decrease both the values of $\alpha$ and $\beta$ at the same time.

Problem 4. Finally, we consider models (1)-(3) corresponding to the form [38]

$$
\begin{aligned}
\frac{\partial w(x, y, t)}{\partial t} & =\left(D_{0 t}^{(1-\alpha)}+D_{0 t}^{(1-\beta)}\right)\left[\frac{\partial^{2} w(x, y, t)}{\partial x^{2}}+\frac{\partial^{2} w(x, y, t)}{\partial y^{2}}\right]+f(x, t), \quad 0<t \leq 1,0<x, y<1, \\
f(x, y, t) & =\exp \left(-\frac{(x-0.5)^{2}}{\gamma}-\frac{(y-0.5)^{2}}{\gamma}\right)\left[f_{1}(x, y, t)+f_{2}(x, y, t)\right],
\end{aligned}
$$


TABLe 2: Numerical results using the proposed numerical scheme corresponding to Problem 2.

\begin{tabular}{lccc}
\hline$M=80, n=9$ & & $\alpha=0.5, \beta=0.5$ \\
\hline$N$ & $L_{\infty}$ & $L_{2}$ & $\kappa$ \\
\hline 11 & $5.4148 \mathrm{e}-005$ & $1.2209 \mathrm{e}-004$ & $5.0986 \mathrm{e}+033$ \\
21 & $1.3432 \mathrm{e}-004$ & $2.7509 \mathrm{e}-004$ & $5.0985 \mathrm{e}+033$ \\
31 & $1.1372 \mathrm{e}-004$ & $3.2761 \mathrm{e}-004$ & $5.0985 \mathrm{e}+033$ \\
41 & $8.6091 \mathrm{e}-005$ & $3.1340 \mathrm{e}-004$ & $5.0985 \mathrm{e}+033$ \\
51 & $1.2453 \mathrm{e}-004$ & $5.3029 \mathrm{e}-004$ & $5.0985 \mathrm{e}+033$ \\
71 & $4.5906 \mathrm{e}-005$ & $2.1446 \mathrm{e}-004$ & $\kappa$ \\
\hline$M=80, n=9, N=41$ & $L_{\infty}$ & $L_{2}$ & $5.1040 \mathrm{e}+033$ \\
$(\alpha, \beta)=(0.2,0.6)$ & 0.0072 & 0.0331 & $5.1040 \mathrm{e}+033$ \\
$(\alpha, \beta)=(0.2,0.4)$ & 0.0019 & 0.0089 & $2.6797 \mathrm{e}+035$ \\
$(\alpha, \beta)=(0.2,0.1)$ & $5.1121 \mathrm{e}-004$ & 0.0023 & $5.0985 \mathrm{e}+033$ \\
$(\alpha, \beta)=(0.6,0.3)$ & 0.0039 & 0.0179 & $5.0985 \mathrm{e}+033$ \\
$(\alpha, \beta)=(0.4,0.3)$ & $4.3443 \mathrm{e}-004$ & 0.0019 & $2.1983 \mathrm{e}+034$ \\
$(\alpha, \beta)=(0.1,0.3)$ & 0.0021 & 0.0096 & \\
\hline
\end{tabular}
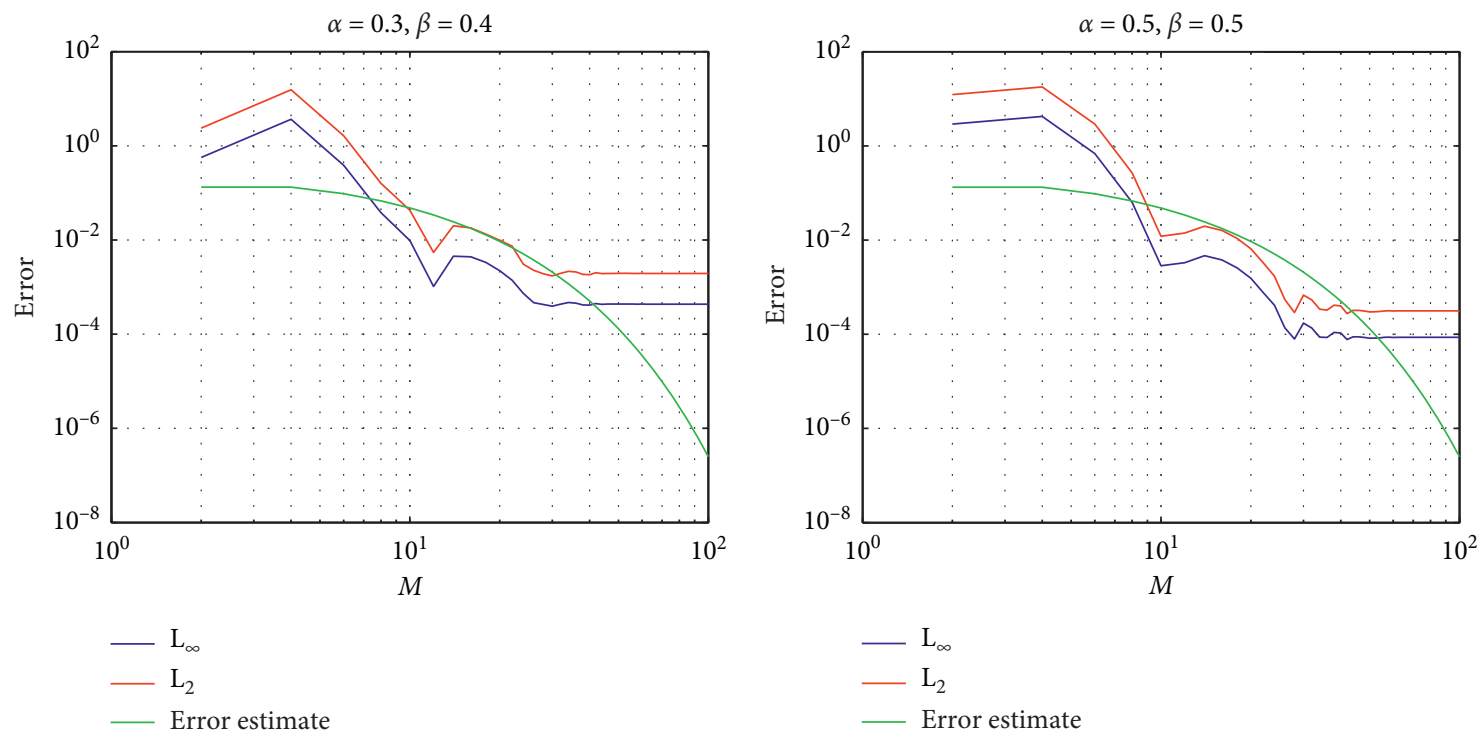

(a)

(b)

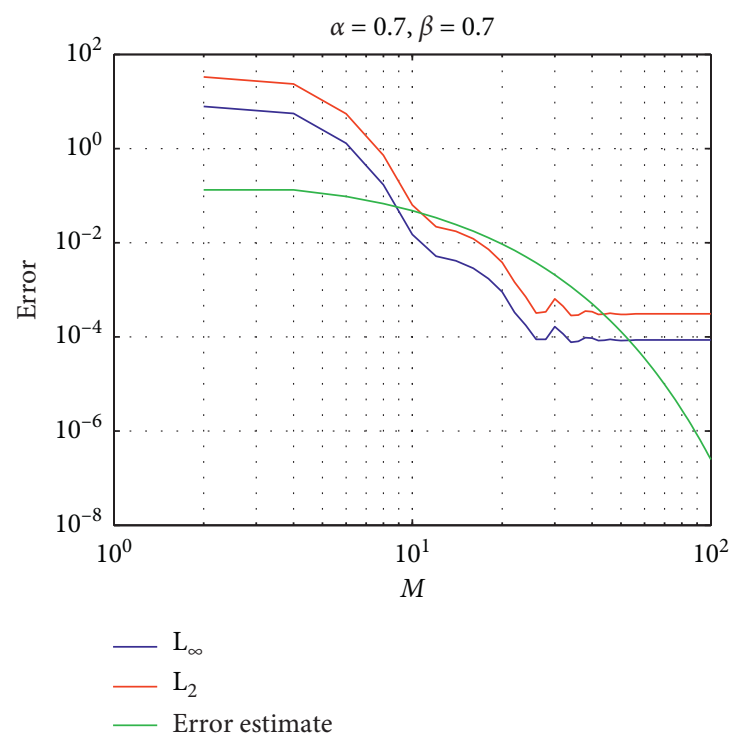

(c)

Figure 2: Error versus quadrature points $M$ at $N=41, n=9$, and $t=1$ and various values of $\alpha$, $\beta$ corresponding to Problem 2 . 
TABLE 3: Numerical results using the proposed numerical scheme corresponding to Problem 3.

\begin{tabular}{lccr}
\hline$M=50, n=11$ & \multicolumn{2}{c}{$\alpha=0.5, \beta=0.5$} \\
\hline$N$ & $L_{\infty}$ & $L_{2}$ & $\kappa$ \\
\hline 15 & $7.3893 \mathrm{e}-004$ & 0.0050 & $3.1023 \mathrm{e}+020$ \\
20 & 0.0025 & 0.0232 & $3.1024 \mathrm{e}+020$ \\
26 & 0.0029 & 0.0344 & $3.0989 \mathrm{e}+020$ \\
41 & $8.6091 \mathrm{e}-005$ & $3.1340 \mathrm{e}-004$ & $5.0985 \mathrm{e}+033$ \\
\hline$M=50, n=15, N=20$ & $L_{\infty}$ & $L_{2}$ & $\kappa$ \\
\hline$(\alpha, \beta)=(0.6,0.5)$ & $2.2399 \mathrm{e}-004$ & 0.0017 & $3.1000 \mathrm{e}+020$ \\
$(\alpha, \beta)=(0.5,0.3)$ & $2.2409 \mathrm{e}-004$ & 0.0017 & $5.0520 \mathrm{e}+021$ \\
$(\alpha, \beta)=(0.3,0.2)$ & $2.2419 \mathrm{e}-004$ & 0.0017 & $1.1756 \mathrm{e}+024$ \\
\hline
\end{tabular}

TABLE 4: Numerical results using the proposed numerical scheme corresponding to Problem 4.

\begin{tabular}{lccr}
\hline$M=50, n=9$ & \multicolumn{2}{c}{$\alpha=0.5, \beta=0.3, \gamma=0.2$} & $\kappa$ \\
\hline$N$ & $L_{\infty}$ & 0.0249 & $1.3362 \mathrm{e}+021$ \\
11 & 0.0069 & 0.0102 & $2.7478 \mathrm{e}+021$ \\
15 & 0.0022 & 0.0057 & $6.7578 \mathrm{e}+021$ \\
21 & $4.4497 \mathrm{e}-004$ & 0.0112 & $1.3745 \mathrm{e}+022$ \\
25 & $7.8109 \mathrm{e}-004$ & $L_{2}$ & $\kappa$ \\
\hline$M=50, N=20, n=11$ & $L_{\infty}$ & 0.0060 & $9.6487 \mathrm{e}+025$ \\
$(\alpha, \beta)=(0.2,0.2)$ & $6.2477 \mathrm{e}-004$ & 0.0060 & $3.1021 \mathrm{e}+020$ \\
$(\alpha, \beta)=(0.5,0.5)$ & $6.2141 \mathrm{e}-004$ & 0.0059 & $3.0989 \mathrm{e}+020$ \\
$(\alpha, \beta)=(0.7,0.7)$ & $6.1740 \mathrm{e}-004$ & 0.0058 & $3.0989 \mathrm{e}+020$ \\
$(\alpha, \beta)=(0.9,0.9)$ & $6.1134 \mathrm{e}-004$ & & \\
\hline
\end{tabular}

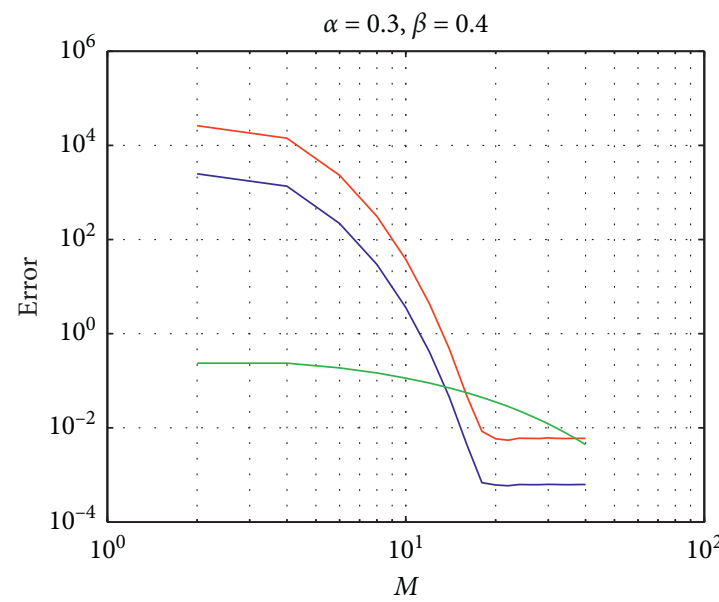

$-\mathrm{L}_{\infty}$

$-\mathrm{L}_{2}$

_ Error estimate

(a)

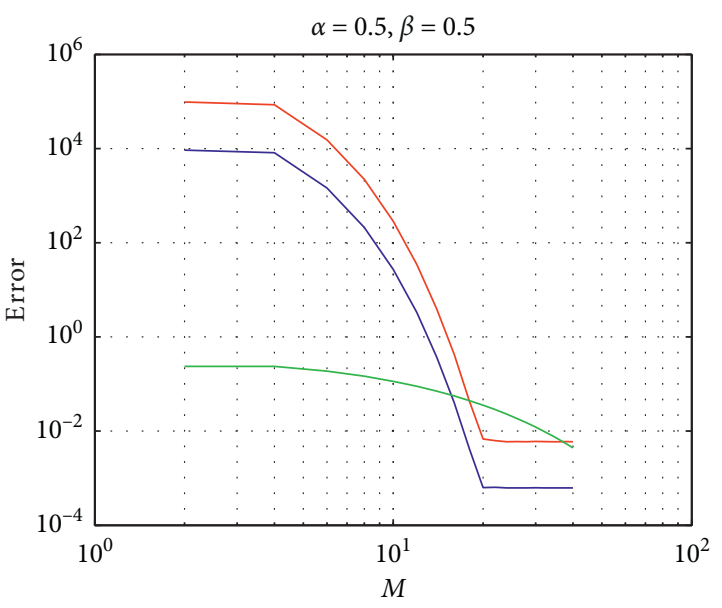

$-\mathrm{L}_{\infty}$

$-\mathrm{L}_{2}$

_ Error estimate

FIgURE 3: Error versus quadrature points $M$ at $N=20, n=11$, and $t=1$ and various values of $\alpha, \beta$ corresponding to Problem 4 . 
where

$$
\begin{aligned}
& f_{1}(x, y, t)=(1+\alpha+\beta) t^{\alpha+\beta}+2 \frac{\Gamma(2+\alpha+\beta)}{\Gamma(1+2 \alpha+\beta)} t^{2 \alpha+\beta} \frac{2}{\gamma}+2 \frac{\Gamma(2+\alpha+\beta)}{\Gamma(1+\alpha+2 \beta)} t^{\alpha+2 \beta} \frac{2}{\gamma}, \\
& f_{2}(x, y, t)=-4\left(\frac{\Gamma(2+\alpha+\beta)}{\Gamma(1+2 \alpha+\beta)} t^{2 \alpha+\beta}+\frac{\Gamma(2+\alpha+\beta)}{\Gamma(1+\alpha+2 \beta)} t^{\alpha+2 \beta}\right)\left(\frac{(x-0.5)^{2}}{\gamma^{2}}+\frac{(y-0.5)^{2}}{\gamma^{2}}\right) .
\end{aligned}
$$

The exact solution is

$$
w(x, y, t)=t^{1+\alpha+\beta} \exp \left(-\frac{(x-0.5)^{2}}{\gamma}-\frac{(y-0.5)^{2}}{\gamma}\right) \text {. }
$$

Here, the problem is solved over the domain $\Omega=[0,1] \times[0,1]$. In the upper section of Table 4 , the $\ell_{\infty}$ and $\ell_{2}$ error norms are decreasing with $\alpha=0.5, \beta=0.3$, $n=9$, and $\gamma=0.2$ and for nodal points $11 \leq N \leq 25$. In the lower section of Table 4 , for same values of $\alpha$ and $\beta$, the $\ell_{\infty}$ and $\ell_{2}$ error norms are decreasing steadily at $N=20, n=11$, and $M=50$. The results are comparatively identical with the results of the paper [38]. Figure 3 shows the error with varying quadrature points $M$ and various values of $\alpha$ and $\beta$ at $N=20, M=50$, and $\gamma=0.2$. The error $L_{\infty}$ is well matched with estimate $L_{\text {est }}$ for $c=0.5$ and $L_{2}$ error norm, as shown in Figure 3. The present method is stable and accurate in multidimensional fractional order partial differential equations.

\section{Conclusion}

In this work, a numerical scheme is constructed which is based on Laplace transform and radial basis functions in the local setting. The proposed numerical scheme efficiently approximated time fractional anomalous subdiffusion equation. The supremacy of this method particularly for fractional order equations is its nonsensitive nature in time as contrary to finite difference approximation for fractional order operators. Since the fractional order derivative is of integral convolution type and suited to handle by Laplace transform, the spatial operators in multidimensions can be approximated by RBF in the local setting which generates small size differentiation matrices in local subdomains and these are assembled as a single sparse matrix in the global domain. So, large amount of data can be manipulated very easily and accurately.

\section{Data Availability}

The data supporting the results are available within the article.

\section{Conflicts of Interest}

The authors declare that they have no conflicts of interest.

\section{References}

[1] K. S. Miller and B. Ross, An Introductional the Fractional Calculus and Fractional Differential Equations, John Wiley \& Sons, Inc., New York, NY, USA and London, UK, 1974.

[2] A. Mohebbi and M. Dehghan, "The use of compact boundary value method for the solution of two-dimensional Schrödinger equation," Journal of Computational and Applied Mathematics, vol. 225, no. 1, pp. 124-134, 2009.

[3] K. B. Oldham and J. Spanier, The Fractional Calculus, Academic Press, London, UK, 1974.

[4] K. Diethelm and N. J. Ford, "Analysis of fractional differential equations," Journal of Mathematical Analysis and Applications, vol. 265, no. 2, pp. 229-248, 2002.

[5] O. Keith and J. Spanier, The Fractional Calculus Theory and Applications of Differentiation and Integration to Arbitrary Order, Elsevier, Amsterdam, Netherlands, 1974.

[6] A. T. Patera, "A spectral element method for fluid dynamics: laminar flow in a channel expansion," Journal of Computational Physics, vol. 54, no. 3, pp. 468-488, 1984.

[7] I. Podlubny, Fractional Differential Equations: An Introduction to Fractional Derivatives, Fractional Differential Equations, to Methods of Their Solution and Some of Their Applications, Elsevier, Amsterdam, Netherlands, 1998.

[8] R. L. Bagley and P. J. Torvik, "A theoretical basis for the application of fractional calculus to viscoelasticity," Journal of Rheology, vol. 27, no. 3, pp. 201-210, 2010.

[9] F. Mainardi, "Fractional calculus," Fractals and Fractional Calculus in Continuum Mechanics, Springer, Berlin, Germany, pp. 291-348, 1997.

[10] Y. A. Rossikhin and M. V. Shitikova, "Applications of fractional calculus to dynamic problems of linear and nonlinear hereditary mechanics of solids," Applied Mechanics Reviews, vol. 50, no. 1, pp. 15-67, 1997.

[11] R. T. Baillie, "Long memory processes and fractional integration in econometrics," Journal of Econometrics, vol. 73, no. 1, pp. 5-59, 1996.

[12] S. Larsson, M. Racheva, and F. Saedpanah, "Discontinuous Galerkin method for an integro-differential equation modeling dynamic fractional order viscoelasticity," Computer Methods in Applied Mechanics and Engineering, vol. 83, pp. 196-209, 2015.

[13] J. T. Machado, V. Kiryakova, and F. Mainardi, "Recent history of fractional calculus," Communications in Nonlinear Science and Numerical Simulation, vol. 16, no. 3, pp. 1140-1153, 2011.

[14] R. Hilfer, Applications of Fractional Calculus in Physics, World Scientific, Singapore, 2000.

[15] R. Amirov, A. Ergun, and S. Durak, "Half-inverse problems for the quadratic pencil of the Sturm-Liouville equations with impulse," Numerical Methods for Partial Differential Equations, vol. 37, no. 1, pp. 915-924, 2021. 
[16] E. R. G. Abdullah, "The multiplicity of eigenvalues of a vectorial singular diffusion equation with discontinuous conditions," Eastern Anatolian Journal of Science, vol. 6, no. 2, pp. 22-34, 2019.

[17] A. Rauf and E. Abdullah, "Half inverse problems for the impulsive singular diffusion operator," Turkish Journal of Science, vol. 5, no. 3, pp. 186-198, 2020.

[18] M. J. Saxton, "Anomalous subdiffusion in fluorescence photobleaching recovery: a Monte Carlo study," Biophysical Journal, vol. 81, no. 4, pp. 2226-2240, 2001.

[19] R. Metzler and J. Klafter, "The random walk's guide to anomalous diffusion: a fractional dynamics approach," Physics Reports, vol. 339, no. 1, pp. 1-77, 2000.

[20] G. M. Zaslavsky, "Fractional kinetic equation for Hamiltonian chaos," Physica D: Nonlinear Phenomena, vol. 76, no. 1-3, pp. 110-122, 1994.

[21] R. Metzler and J. Klafter, "The restaurant at the end of the random walk: recent developments in the description of anomalous transport by fractional dynamics," Journal of Physics A: Mathematical and General, vol. 37, no. 31, pp. R161-R208, 2004.

[22] M. Dehghan and A. Nikpour, "The solitary wave solution of coupled Klein-Gordon-Zakharov equations via two different numerical methods," Computer Physics Communications, vol. 184, no. 9, pp. 2145-2158, 2013.

[23] M. Dehghan and A. Shokri, "Numerical solution of the nonlinear Klein-Gordon equation using radial basis functions," Journal of Computational and Applied Mathematics, vol. 230, no. 2, pp. 400-410, 2009.

[24] M. Tatari and M. Dehghan, "On the solution of the non-local parabolic partial differential equations via radial basis functions," Applied Mathematical Modelling, vol. 33, no. 3, pp. 1729-1738, 2009.

[25] Y. T. Gu, P. Zhuang, and F. Liu, "An advanced implicit meshless approach for the non-linear anomalous subdiffusion equation," Computer Modeling in Engineering \& Sciences, vol. 56, no. 3, pp. 303-334, 2010.

[26] L. Ling and R. Schaback, "Stable and convergent unsymmetric meshless collocation methods," SIAM Journal on Numerical Analysis, vol. 46, no. 3, pp. 1097-1115, 2008.

[27] R. Schaback, "Convergence of unsymmetric kernel-based meshless collocation methods," SIAM Journal on Numerical Analysis, vol. 45, no. 1, pp. 333-351, 2007.

[28] E. J. Kansa and Y. C. Hon, "Circumventing the ill-conditioning problem with multiquadric radial basis functions: applications to elliptic partial differential equations," Computers \& Mathematics with Applications, vol. 39, no. 7-8, pp. 123-137, 2000.

[29] B. Šarler, "A radial basis function collocation approach in computational fluid dynamics," Computer Modelling in Engineering \& Sciences, vol. 7, pp. 185-193, 2005.

[30] B. Šarler, "Meshfree explicit local radial basis function collocation method for microscopic and macroscopic phase change simulations," $\mathrm{PhD}$ Thesis, Univerza v Novi Gorici, Nova Gorica, Slovenia, 2006.

[31] V. Voller, R. Vertnik, and B. Šarler, "Meshless local radial basis function collocation method for convective-diffusive solid-liquid phase change problems," International Journal of Numerical Methods for Heat \& Fluid Flow, vol. 16, no. 5, 2006.

[32] Q. T. L. Gia and W. McLean, "Solving the heat equation on the unit sphere via laplace transforms and radial basis functions," Advances in Computational Mathematics, vol. 40, no. 2, pp. 353-375, 2014.
[33] G. J. Moridis and E. Kansa, The Laplace Transform Multiquadric Method: A Highly Accurate Scheme for the Numerical Solution of Partial Differential Equations, Lawrence Berkeley National Laboratory, Berkeley, CF, USA, 1993.

[34] M. López-Fernández and C. Palencia, "On the numerical inversion of the laplace transform of certain holomorphic mappings," Applied Numerical Mathematics, vol. 51, no. 2-3, pp. 289-303, 2004.

[35] B. A. Jacobs, "High-order compact finite difference and laplace transform method for the solution of time-fractional heat equations with dirchlet and neumann boundary conditions," Numerical Methods for Partial Differential Equations, vol. 32, no. 4, pp. 1184-1199, 2016.

[36] W. McLean and V. Thomée, "Time discretization of an evolution equation via laplace transforms," IMA Journal of Numerical Analysis, vol. 24, no. 3, pp. 439-463, 2004.

[37] W. McLean and V. Thomée, "Numerical solution via laplace transforms of a fractional order evolution equation," Journal of Integral Equations Applications, vol. 22, no. 1, pp. 57-94, 2010.

[38] M. Dehghan, M. Abbaszadeh, and A. Mohebbi, "Legendre spectral element method for solving time fractional modified anomalous sub-diffusion equation," Applied Mathematical Modelling, vol. 40, no. 5-6, pp. 3635-3654, 2016.

[39] K. S. Miller and B. Ross, An Introduction to the Fractional Calculus and Fractional Differential Equations, John Wiley \& Sons, New York, NY, USA, 1993.

[40] I. Podlubny, "Fractional differential equations," Mathematics in Science and Engineering, Vol. 198, Academic Press, Cambridge, MA, USA, 1999.

[41] A. M. Cohen, Numerical Methods for Laplace Transform Inversion, Springer Science+Business Media, LLC, Berlin, Germany, 2007.

[42] M. Uddin, H. Ali, and A. Ali, "Kernel-based local meshless method for solving multi-dimensional wave equations in irregular domain," CMES-Computer Modeling in Engineering \& Sciences, vol. 107, no. 6, pp. 463-479, 2015.

[43] M. Uddin, Z. Minullah, and A. Ali, "On the approximation of rapidly oscillatory hankel transform via radial kernels," Dolomites Research Notes on Approximation, vol. 11, no. 1, 2018.

[44] N. Zhang, W. Deng, and Y. Wu, "Finite difference/element method for a two-dimensional modified fractional diffusion equation," Advances in Applied Mathematics and Mechanics, vol. 4, no. 4, pp. 496-518, 2012. 